\title{
Employee Reactions to Interruptions from Family during Work
}

\author{
Michael Horvath $^{1}$ (D) Jacqueline S. Gueulette ${ }^{1,2} \cdot$ Kiara A. Gray $^{1,3}$
}

Received: 3 October 2019 / Revised: 11 December 2020 / Accepted: 9 February 2021 / Published online: 27 March 2021

(C) The Author(s), under exclusive licence to Springer Nature Switzerland AG part of Springer Nature 2021

\begin{abstract}
In contemporary workplaces, spouses, parents, and children are frequently able to reach employees through telephone, text, or email. Although some employees may welcome this level of accessibility to their families, others may react negatively, but we know little about the individual differences or situational characteristics that relate to these reactions. To examine these reactions, we recruited samples of US residents, employed outside the home in a variety of occupations, who reported that they were recently interrupted at work by a family member. We measured four reactions to the episode (anger, sadness, complaining about the interruption, and the degree to which the employee forgave the family member). Results showed reactions related to interactions between two features of the interruption (the importance of the interruption and the severity of the disruption to one's work) and the employee's preference for integrating family and work roles.
\end{abstract}

Keywords Work-family balance $\cdot$ Boundary management $\cdot$ Interruptions

In recent decades, the workplace has been typically separated from one's family. Although it was possible to contact family members at work, most interactions with family members waited until employees returned home. However, this line may be dissolving in many workplaces. Family members now have greater ability to contact each other at work through technologies such as email and mobile devices. Furthermore, the increase in practices such as telecommuting permit even greater access to

An early version of this research was presented at the $35^{\text {th }}$ Annual Conference of the Society for Industrial and Organizational Psychology.

Michael Horvath

m.horvath59@csuohio.edu

1 Department of Psychology, Cleveland State University, 2121 Euclid Avenue, Cleveland, $\mathrm{OH} 44115$, USA

2 Bologna Business School, University of Bologna, Bologna, Italy

3 Department of Psychology, Xavier University, Cincinnati, OH, USA 
employees during work hours - a trend that has been dramatically exacerbated by the COVID-19 pandemic, and particularly for families with children.

The increased ability to communicate with family members, either through technology or proximity, is likely to increase the extent to which family members are interrupted while they are working, potentially increasing perceptions of conflict between one's work and family roles. Therefore, it is important to understand the impact of interruptions. How do employees react to interruptions from family? Are all types of interruptions equally detrimental? Do some individuals tolerate interruptions better than others?

Although the work-family conflict literature suggests that overall perceptions of conflict may aggregate from discrete instances of interruptions and other boundary intrusions, few studies have examined the impact of a specific interruption from family at work. Furthermore, the existing studies have examined only a limited range of employee reactions, and have not examined how situational factors and individual differences may work in conjunction to influence these reactions. Our study extends existing research by focusing on reactions to a single interruption, examining how two characteristics of the interruption - its importance and severity - interact with employees' boundary management strategies.

\section{Work-Family Conflict and Boundary Management}

Individuals engage in many life domains such as work and family (e.g., Greenhaus \& Beutell, 1985). They may devote differing amounts of time to each domain, and may enact different schemas or roles within each of those domains. These roles or time demands may not always be compatible with each other. For instance, one might be forced to choose between spending time with family in the evening or returning to one's home office to finish a project. Competition between work and family roles is typically described as work-family conflict (WFC). WFC is not a unidimensional phenomenon. Types of conflict include issues of time, of different behaviors that are effective in one domain but not another, or even a spillover of strains from one domain into another. Furthermore, Frone et al. (1992) clarify the importance of the direction of the relationship. Work roles can interfere with family responsibilities (WIF) to a different extent than family interferes with work (FIW). WFC has been linked to a host of outcomes including job and family satisfaction and other strains (see numerous meta-analyses including Allen et al., 2020; Michel et al., 2009; Nohe et al., 2015).

To help manage potential conflicts between work and non-work roles, individuals establish and maintain psychological boundaries between the domains (e.g., Ashforth et al., 2000; Clark, 2000; Nippert-Eng, 1996). These boundaries can vary in both permeability and flexibility. Permeable boundaries allow elements of one domain to be present in the other (e.g., family communications during work time), and flexible boundaries allow work to be rearranged in order to accommodate demands of another domain (e.g., leaving work early to care for a family member while making up the time later). The degrees of permeability and flexibility may be arranged along a continuum from integration (in which elements of work and family mix freely) to segmentation (in which each domain is kept as separate as possible). Furthermore, like WFC, boundary management may be asymmetrical (Ashforth et al., 2000); one domain may be 
protected from intrusions more than another. In our study, we focus on permeability the extent to which communications from family members enter one's work role.

Permeable boundaries imply that one domain may intrude upon another. It is likely that regardless of the domain, an individual would be engaged in domain-related activity when such an intrusion occurs. For instance, although it is possible that family members call or text while the employee is on a work break, in many workplaces it is also likely that such communications may occur when the individual is engaged in a work task. Therefore, permeability leads to workplace interruptions. Below, we briefly discuss the literature on task interruptions, and more specifically the cross-domain interruptions known as boundary violations.

\section{Task Interruptions, Intrusions, and Boundary Violations}

A variety of interruptions permeate the modern workplace. Jett and George (2003) differentiated between four primary types of interruptions: intrusions, breaks, distractions, and discrepancies. We focus on the intrusion, defined as "an unexpected encounter initiated by another person that interrupts the flow and continuity of an individual's work and brings that work to a temporary halt" (p. 495). Within the realm of border theory, an intrusion from one domain into another (e.g., family contacting an employee during work) is described as a "boundary violation."

Jett and George (2003) described a number of reasons why intrusions may lead to negative outcomes for individuals and organizations. For instance, the amount of time required to address the intrusion reduces the amount of time that may be available to complete the primary task. Consequently, interrupted employees may experience increased anxiety and stress. Furthermore, switching between tasks may limit the extent to which one can become fully immersed in the target task, reducing engagement and "flow." Baethge and Rigotti's (2013) review of previous literature on interruptions highlighted several other reasons to expect intrusions to have negative effects. For instance, the interruption may result in the individual forgetting to complete the original task unless additional effort is devoted to rehearsing one's intention to resume the task. Switching tasks also require additional cognitive resources, particularly if the knowledge and skill requirements for the primary task and the interrupting task differ. Finally, in order to compensate for the loss of time, individuals may decrease both the quantity and the quality of their work when they return to the original task. Such loss may occur in part due to the effects of 'attention residue,' whereby rumination about a previous task impairs performance on the next task (e.g., Newton et al., 2020).

Empirical research has provided support for the proposed negative effects of intrusions. For instance, Baethge and Rigotti (2013) found that interruptions related to mental demands, time pressure, reduced (forgotten) intentions to complete the primary task, and irritation. Pachler et al. (2018) related interruptions to outcomes such as increased stress, time needed to complete the primary task, emotional exhaustion, and satisfaction with performance. Wilkes et al. (2018) tied their measure of intrusions to outcomes like stress, exhaustion, and interpersonal conflict. More specific to the present study, Hunter et al. (2019) related boundary violations to work goal obstruction, which led to negative affect at work, which in turn led to family-to-work conflict. 
Jett and George (2003) also discussed the possibility that intrusions have positive effects at times. For instance, interruptions may result in improved communication, higher quality relationships, and improving coworkers' performance (e.g., if the intrusion involved a coworker seeking help or knowledge). This perspective is a reminder that individuals rarely adopt a single goal in isolation. Although individuals may focus on one goal at a time, they may have multiple tasks to accomplish in a given day at work, and furthermore must remain aware of the goals that they hold for other roles (e.g., spouse or parent). Thus, an intrusion or boundary violation may hinder one goal, but it may also facilitate the attainment of other goals. For instance, Hunter et al. (2019) also found that boundary violations at work led to a greater sense of family goal facilitation, which then related to positive affect.

\section{Reactions to Interruptions}

Employees may experience a host of reactions to an interruption, but we chose to focus on four - two affective and two behavioral. First, consistent with recent calls to investigate emotional reactions to boundary violations (e.g., Hunter et al., 2019; Newton et al., 2020), we explored two affective reactions. Anger (or closely related emotions such as agitation and frustration; also see Baethge \& Rigotti's 2013, relationship between interruptions and irritation) may be common. Anger may have important implications both for family functioning (e.g., if the anger is expressed to family members in maladaptive ways) as well as for the workplace, as affective reactions may color subsequent job performance or interactions with coworkers (e.g., Beal et al., 2005). Similarly, we examined potential effects on sadness (e.g., violations may result in a state of helplessness). Third, we examined the extent to which employees complained or vented to others. Complaining to the interrupting party may be one way to communicate and manage one's boundaries. Furthermore, complaining to others may be a way to seek emotional support such as empathy, a common coping mechanism for workplace stressors (e.g., Viswesvaran et al., 1999). Finally, we examined the extent to which employees forgave their family member for the interruption. Forgiveness has been related to positive outcomes like greater life satisfaction and lower depression and anxiety, as well as to job performance and employee morale (Madsen et al., 2009; Riek \& Mania, 2012), and as such is an important outcome to study.

\section{Characteristics of Boundary Violations}

As shown above, intrusions and boundary violations may generate negative reactions, although this may not always be the case. When will a violation be perceived more negatively? Wilkes et al. (2018) speculated that characteristics related to particular interruptions may influence outcomes. Pachler et al. (2018) noted the dearth of studies that explored different types of interruptions and called for more research. Therefore, below, we extend this line of research by examining two characteristics of interruptions that we believe may influence one's reactions. 


\section{Interruption Importance}

First, we expected that the importance of the interruption would generate more positive reactions (i.e., lower sadness, anger, and complaining, as well as higher forgiveness). Some interruptions legitimately require the employee to respond during work hours (e.g., a family emergency), whereas other interruptions are less important or might have waited until after work (e.g., helping a child with a homework problem). Hunter et al. (2019) argued that individuals are motivated to satisfy goals in multiple life domains (e.g., work and family). This argument reflects theories of multiple-goal self-regulation (e.g., Sun \& Frese, 2013) that discuss how individuals allocate their time and attention across multiple goals, by incorporating the role of each goal's valence or importance. For instance, Schmidt et al. (2009) found that individuals allocated more resources to goals with more deficient progress. From the perspective of a boundary intrusion, an important family goal may be one that cannot wait until work is over, thereby increasing the perceived discrepancy between the current state and the desired state of that domain. Consequently, we hypothesize the following:

Hypothesis 1: The importance of the interruption is related to more positive reactions to the interruption (i.e., lower anger, sadness, and complaining, but higher forgiveness).

\section{Interruption Severity}

Interruptions also differ in severity. For instance, some interruptions may require considerable time (e.g., leaving work to drive home) or cognitive effort (e.g., helping a child with a homework problem on differential calculus). Furthermore, severity may also relate to the task being interrupted. For instance, if one's work task was cognitively demanding, it may require greater time to reengage in the task once one is able to resume work. Lee and Duffy (2015) found that when the primary task was cognitively demanding, it took greater time to complete following an interruption - particularly if the interruption was also cognitively demanding. While not studying interruptions specifically, Smit et al. (2016) found that cognitively transitioning between work and family roles led to increased self-regulatory depletion, which in turn reduced job performance. Conservation of Resources Theory (e.g., Hobfoll, 1989) argues that individuals are motivated to acquire and protect their resources. Thus, the potentially deleterious effect of interruptions on cognitive and temporal resources should lead interrupted employees to experience more negative reactions (i.e., increased anger, sadness, and complaining, as well as lower forgiveness):

Hypothesis 2: The severity of the interruption is related to more negative reactions to the interruption (i.e., higher anger, sadness, and complaining, but lower forgiveness).

Furthermore, the importance of the interruption may buffer the impact of severity. That is, as the valence of the family goal increases (perhaps leading to a decrease in the 
relative importance of the primary task), an employee may be more amenable to expending greater resources on that goal regardless of the cost to the primary task.

Hypothesis 3: Interruption importance will moderate the relationship between severity and reactions, such that the effect of severity will be weaker for more important interruptions.

\section{Boundary Management Preferences}

Several authors (e.g., Hunter et al., 2019; Pachler et al., 2018; Wilkes et al., 2018) have recently called for an increased investigation of individual difference factors related to workplace interruptions. Earlier, we discussed how boundary management can be arrayed on a continuum from integration (allowing permeable boundaries between domains) to segmentation (keeping the domains separate), and we believe that boundary management styles play an important role in reactions to interruptions.

Hunter et al. (2019) proposed that integrators would not be as affected by boundary violations given their familiarity with, and perhaps even preferences for, such interruptions. This view is supported by Smit et al. (2016), who found that the relationship between cognitive role transitions and resource depletion was weaker for integrators than for segmenters. Although both of these studies appeared to address typical, or enacted, boundary management styles, it is important to recognize that one's actual boundary management may differ from one's preferences for boundary management (e.g., Kossek \& Lautsch, 2012; Kreiner et al., 2009). We propose that individuals who prefer segmenting will react more negatively to boundary violations than those who prefer integrating. That is, they will experience more anger and sadness, and will complain more and forgive less. Furthermore, consistent with Hunter et al. and Smit et al. we believe that individuals who prefer to integrate will tend to have positive reactions to boundary violations regardless of the interruption's severity or importance, as interruptions are relatively expected and welcome. However, those who prefer segmentation may see an interruption as a violation of their expectations, which may lead to greater cognitive processing of the reasons behind the interruption. In other words, individuals who prefer segmentation may view a boundary violation as a threat during a primary appraisal process (e.g., Lazarus, 1966), breaking out of automatic processes and initiating subsequent cognition. Thus, these individuals may be more likely to evaluate the features of the interruption and subsequently experience an interaction between them.

Hypothesis 4: Individuals who prefer segmentation will react more negatively to an interruption (i.e., higher anger, sadness, and complaining, but lower forgiveness) than individuals who prefer integration.

Hypothesis 5: There will be a three-way interaction between boundary management preferences, interruption importance, and interruption severity, such that the two-way interaction between importance and severity will be more prominent for individuals who prefer segmentation. 
In summary, Hunter et al. (2019, p. 1285) wrote that "a focus on the process linking boundary violation events to outcomes is critical, because individuals may experience very different outcomes based on the extent to which they appraise the boundary violation events positively or negatively." Our research advances our understanding of these processes. Understanding how the features of boundary violations affect reactions will allow for more structured approaches to boundary management. Knowing whether certain individuals are more likely to be influenced by boundary violation features can also lead to new processes such as improved communication about specific issues between employees and their families.

\section{Method}

\section{Participants}

Our data collection consisted of two samples (the first in the summer of 2018 and the second in February 2020). We drew both samples from Amazon's Mechanical Turk (MTurk), using MTurk IDs to prevent duplicate participants. Participants from the first sample received $\$ .25$ and participants from the second sample were compensated $\$ 1.21 .^{1}$

We recruited 364 total participants. Sixty-five were eliminated based on "attention check" questions and 102 were eliminated for not following given instructions (see Procedures below), for a total usable sample size of 197. Out of the participants who responded to demographic questions, $51.0 \%$ were women. The age of participants ranged from 20 to 67 years old $(M=36.55, S D=10.91)$. With regard to race, $84.8 \%$ indicated being White/Caucasian, 6.6\% were Black/African American, 6.6\% were Hispanic/Latino, $4.6 \%$ were Asian, and .5\% were American Indian. As far as education level, .8\% of participants had some high school, $10.7 \%$ were high school graduates, $20.3 \%$ had some college but no degree, $12.2 \%$ had an associate's degree, $47.7 \%$ had a bachelor's degree, and $8.6 \%$ had a postgraduate degree. The relationship status of the participants are as follows: $54.8 \%$ were married, $1.0 \%$ were widowed, $5.6 \%$ were divorced, $1.5 \%$ were separated, $5.6 \%$ were in a domestic partnership or civil union, $9.6 \%$ were single but cohabiting with a significant other, and $21.3 \%$ were single. When asked if they have children, $52.6 \%$ of participants responded that they did. Participants worked between 2 to $77 \mathrm{~h}$ per week $(M=38.94, S D=10.59)$, with $21.3 \%$ of participants working from home, $50.3 \%$ of participants not working from home, and $28.4 \%$ of participants sometimes working from home.

\section{Procedure}

Individuals who chose this study from a list of potential tasks on Mechanical Turk were directed to a SurveyMonkey survey. After answering demographic questions, they were asked to focus on "the last time you were contacted by a family member while you were working." They were asked to describe the event, including who interrupted

\footnotetext{
${ }^{1}$ In a previous version of this manuscript a reviewer raised concerns about the level of payment. The compensation in the second sample was thus calibrated to approximate the federal minimum wage.
} 
them and what the interruption was about (not all individuals provided all of this information, but individuals who did not clearly describe a specific interruption were removed from the dataset). Of the individuals who named a specific family member, the most common was the spouse or partner (37\%), followed by parents $(30 \%)$, children (15\%), and siblings (12\%). (Using only these four groups, we did not find that the type of family member related to the focal variables in this study, although some analyses approached significance.) After describing the event, participants responded to our questions regarding this interruption.

\section{Measures $^{2}$}

All measures used 5-point Likert-type scales with anchors ranging from "Strongly Disagree" to "Strongly Agree." Preference for Integration was assessed using five items adapted from Kossek et al. (2012; e.g., "I prefer to take care of personal or family needs at work"). The remaining measures were created for this study. We created five items to assess the interruption's Severity (e.g., "The interruption required a lot of my attention"), four for the interruption's Importance (e.g., "The interruption was important"), four for Anger (e.g., "I was angry"), five for Sadness (e.g., "I was sad"), four for Forgiveness (e.g., "I easily forgave the person who interrupted me"), and six for Complaining (e.g., "I complained about the interruption to the person who interrupted me;" this measure listed a number of potential targets, e.g., the family member, coworkers, etc.).

\section{Measure Validation and Refinement}

We employed multiple strategies to refine and validate the measures that we used. First, we showed each item (in randomized order) to a sample of 14 Masters level students in psychology, asking them to sort each item into the best fitting category (including an option indicating no fit with any category). In general, we eliminated items when fewer than $85 \%$ of the subject matter experts agreed about the item (exceptions were one Importance item, rated $71 \%$ correctly, and many Severity items; for this scale all items were correctly sorted between $64 \%$ and $86 \%$ of the time). We retained all items for boundary management preferences and anger, two items for sadness, four items for complaining, three items for forgiveness, and three items for importance of the interruption. The example items in the previous section were all included.

Next, we subjected the remaining items to an exploratory factor analysis (principal axis factoring with a Promax rotation). Seven factors emerged with eigenvalues greater than one. Furthermore, all items loaded on their intended constructs with no substantial crossloadings with one exception: one of the forgiveness items loaded on the complaining scale. This particular item immediately followed the complaining items on the survey, and was written in the same direction as the complaining items (and reversed from the two other forgiveness items). Thus, we interpret this as a statistical artifact, but recommend that our results be replicated with other scales.

\footnotetext{
${ }^{2}$ We measured several other constructs not reported here that were not related to the hypotheses in this article
} 
Finally, for some of our constructs we were able to locate pre-existing measures of the same construct (i.e., Hostility and Sadness subscales from the PANAS-X, Watson \& Clark, 1994, Venting and Support from the COPE scale, Carver et al., 1989). We administered these measures in our second sample, and given the relatively small sample size we did not use these variables for anything other than convergent validity analyses. Correlations between our measures and these existing measures were strong; for Anger/Hostility, $r=.58, p>.001$, for Sadness, $r=.80, p<.001$, and our Complaining measure correlated .68 with the Venting subscale and .90 with the Support subscale, both $p$ 's $<.001$. Thus, we have additional support for the convergent validity of these scales.

\section{Results}

\section{Descriptive Statistics}

Descriptive statistics can be found in Table 1. With the exception of Forgiveness (.67), all of the dependent variables had internal consistency reliabilities above .85 . The focal variables of this study were rarely (and then, only weakly) related to the demographic variables. Participants in the second sample were more likely to rate the interruption as important, to report working more hours, and to be less likely to be living with a partner, but the samples did not differ on the basis of the other variables in the study.

Analyses of the dependent variables showed substantial deviations from normality, and it was not possible to correct these deviations with a nonlinear transformation. Therefore, we dichotomized each of the dependent variables. For Forgiveness, we differentiated between individuals who reported less than total forgiveness (i.e, less than 5) from those who completely forgave the person who interrupted them (i.e., exactly 5). For the other three variables, we differentiated between individuals who reported absolutely no complaining, anger, or sadness (i.e., exactly 1) from those who reported some amount of these variables (i.e., greater than 1). Table 1 reports both the original and the dichotomized versions of these variables, but our analyses focused on the dichotomized versions.

As shown in Table 1, the importance of the interruption was uncorrelated with all four (dichotomized) dependent variables, but the severity of the interruption related to increased anger, sadness, complaining, and decreased forgiveness. The number of hours worked was correlated significantly with the dichotomized Anger variable, so this variable was included as a covariate when predicting Anger.

\section{Hypothesis Tests}

Given that we tested all four hypotheses for each dependent variable separately, this section is organized by variable rather than by hypothesis. Hierarchical logistic regressions were used for all analyses because the dependent variables were dichotomous. We therefore report the Nagelkerke $R^{2}$ value as an estimate of effect size. Results of the final step of the hierarchical regression for each dependent variable can be found in Tables 2, 3, 4 and 5. 


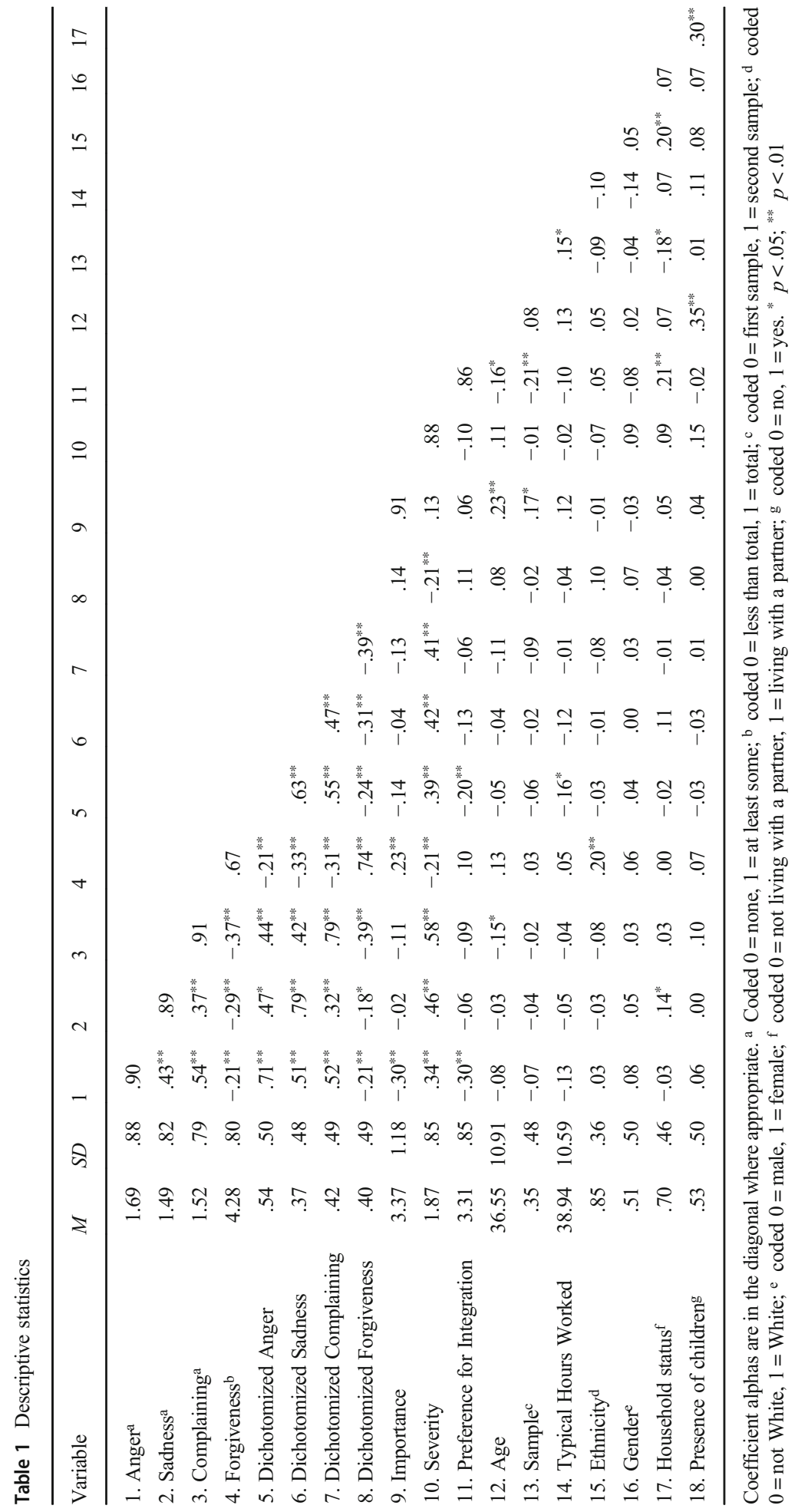


Table 2 Logistic regression of anger on hours worked, integration preferences, importance, and severity

\begin{tabular}{|c|c|c|c|c|c|c|c|}
\hline Variable & $x^{2}$ & $\Delta \chi^{2}$ & $R^{2}$ & $\Delta R^{2}$ & $B$ & $e^{B}$ & $p^{a}$ \\
\hline Step 1 & $5.43^{*}$ & & $.04^{*}$ & $.04^{*}$ & & & .020 \\
\hline Intercept & & & & & $.50^{*}$ & $1.66^{*}$ & .013 \\
\hline Hours Worked & & & & & $-.05^{*}$ & .96 & .011 \\
\hline Step 2 & $53.84^{* *}$ & $48.41^{* *}$ & $.32^{* *}$ & $.28^{* *}$ & & & $<.001$ \\
\hline Importance & & & & & $-.58^{* *}$ & $.56^{* *}$ & $<.001$ \\
\hline Severity & & & & & $1.63^{* *}$ & $5.09^{* *}$ & $<.001$ \\
\hline Preference for Integration & & & & & $-.67^{*}$ & $.51^{*}$ & .021 \\
\hline Step 3 & $58.92^{* *}$ & $5.08^{*}$ & $.35^{* *}$ & $.03^{*}$ & & & $.024^{*}$ \\
\hline Importance $\times$ Severity & & & & & $-.53^{*}$ & $.59^{*}$ & $.024^{*}$ \\
\hline Step 4 & $59.36^{* *}$ & 0.44 & $.35^{* *}$ & .00 & & & .802 \\
\hline Importance $\times$ Preference for Integration & & & & & .18 & 1.20 & .405 \\
\hline Severity $\times$ Preference for Integration & & & & & -.18 & .84 & .676 \\
\hline Step 5 & $59.66^{* *}$ & 0.31 & $.35^{* *}$ & .00 & & & .580 \\
\hline Importance $\times$ Severity $\times$ Preference for Integration & & & & & .16 & 1.17 & .580 \\
\hline
\end{tabular}

Regression coefficients are from Step 5 of the model, and all variables were centered around their means. $\chi^{2}$ values refer to changes in the -2 Loglikelihood value. Nagelkerke $R^{2}$ values are reported for effect size. ${ }^{\text {a }}$ $p$ values refer to the change between models for the rows labeled Step 1, Step 2, etc

* $p<.05 ;{ }^{* *} p<.01$

Table 3 Logistic regression of sadness on integration preferences, importance, and severity

\begin{tabular}{|c|c|c|c|c|c|c|c|}
\hline Variable & $\chi^{2}$ & $\Delta \chi^{2}$ & $R^{2}$ & $\Delta R^{2}$ & $B$ & $e^{B}$ & $p^{a}$ \\
\hline Step 1 & $40.01^{* *}$ & $40.01^{* *}$ & $.25^{* *}$ & $.25^{* *}$ & & & $<.001$ \\
\hline Intercept & & & & & $-.56^{* *}$ & $.57^{* *}$ & $<.001$ \\
\hline Importance & & & & & -.21 & .81 & .180 \\
\hline Severity & & & & & $1.45^{* *}$ & $4.26^{* *}$ & $<.001$ \\
\hline Preference for Integration & & & & & -.22 & .80 & .252 \\
\hline Step 2 & $43.54^{* *}$ & 3.52 & .27 & .02 & & & .061 \\
\hline Importance $\times$ Severity & & & & & $-.45^{*}$ & $.64^{*}$ & .037 \\
\hline Step 3 & $46.74^{* *}$ & 3.21 & .29 & .02 & & & .201 \\
\hline Importance $\times$ Preference for Integration & & & & & -.04 & .96 & .768 \\
\hline Severity $\times$ Preference for Integration & & & & & $.53^{*}$ & $1.69^{*}$ & .031 \\
\hline Step 4 & $47.70^{* *}$ & 0.96 & .29 & .00 & & & .327 \\
\hline Importance $\times$ Severity $\times$ Preference for Integration & & & & & -.17 & .84 & .311 \\
\hline
\end{tabular}

Regression coefficients are from Step 4 of the model, and all variables were centered around their means. $\chi^{2}$ values refer to changes in the -2 Loglikelihood value. Nagelkerke $R^{2}$ values are reported for effect size. ${ }^{\text {a }}$ $p$ values refer to the change between models for the rows labeled Step 1, Step 2, etc

${ }^{*} p<.05 ;{ }^{* *} p<.01$ 
Table 4 Logistic regression of complaining on integration preferences, importance, and severity

\begin{tabular}{|c|c|c|c|c|c|c|c|}
\hline Variable & $\chi^{2}$ & $\Delta \chi^{2}$ & $R^{2}$ & $\Delta R^{2}$ & $B$ & $e^{B}$ & $p^{a}$ \\
\hline Step 1 & $42.87^{* *}$ & $42.87^{* *}$ & $.26^{* *}$ & $.26^{* *}$ & & & $<.001$ \\
\hline Intercept & & & & & -.16 & .85 & .373 \\
\hline Importance & & & & & $-.54^{* *}$ & $.58^{* *}$ & .002 \\
\hline Severity & & & & & $1.68^{* *}$ & $5.35^{* *}$ & $<.001$ \\
\hline Preference for Integration & & & & & .12 & 1.13 & .564 \\
\hline Step 2 & $53.74^{* *}$ & $10.86^{* *}$ & $.32^{* *}$ & $.06^{* *}$ & & & .001 \\
\hline Importance $\times$ Severity & & & & & $-.68^{* *}$ & $.50^{* *}$ & .002 \\
\hline Step 3 & $59.05^{* *}$ & 5.32 & $.35^{* *}$ & .03 & & & .070 \\
\hline Importance $\times$ Preference for Integration & & & & & -.24 & .79 & .132 \\
\hline Severity $\times$ Preference for Integration & & & & & $.64^{*}$ & $1.90^{*}$ & .020 \\
\hline Step 4 & $59.36^{* *}$ & 0.30 & $.35^{* *}$ & .00 & & & .582 \\
\hline Importance $\times$ Severity $\times$ Preference for Integration & & & & & -.11 & .90 & .565 \\
\hline
\end{tabular}

Regression coefficients are from Step 4 of the model, and all variables were centered around their means. $\chi^{2}$ values refer to changes in the -2 Loglikelihood value. Nagelkerke $R^{2}$ values are reported for effect size. ${ }^{\text {a }}$ $p$ values refer to the change between models for the rows labeled Step 1, Step 2, etc

${ }^{*} p<.05 ;{ }^{* *} p<.01$

Table 5 Logistic regression of forgiveness on integration preferences, importance, and severity

\begin{tabular}{|c|c|c|c|c|c|c|c|}
\hline Variable & $\chi^{2}$ & $\Delta \chi^{2}$ & $R^{2}$ & $\Delta R^{2}$ & $B$ & $e^{B}$ & $p$ \\
\hline Step 1 & $16.81^{* *}$ & $16.81^{* *}$ & $.11^{* *}$ & $.11^{* *}$ & & & .001 \\
\hline Intercept & & & & & $-.62^{* *}$ & $.54^{* *}$ & .001 \\
\hline Importance & & & & & $.44^{* *}$ & $1.54^{* *}$ & .006 \\
\hline Severity & & & & & $-.88^{* *}$ & $.42^{* *}$ & .001 \\
\hline Preference for Integration & & & & & .49 & 1.63 & .065 \\
\hline Step 2 & $20.85^{* *}$ & $4.04^{*}$ & $.14^{* *}$ & $.02^{*}$ & & & .045 \\
\hline Importance $\times$ Severity & & & & & $.39^{*}$ & $1.48^{*}$ & .047 \\
\hline Step 3 & $23.55^{* *}$ & 2.70 & $.15^{* *}$ & .01 & & & .259 \\
\hline Importance $\times$ Preference for Integration & & & & & -.38 & .68 & .053 \\
\hline Severity $\times$ Preference for Integration & & & & & .41 & 1.51 & .271 \\
\hline Step 4 & $25.02^{* *}$ & 1.47 & $.16^{* *}$ & .01 & & & .225 \\
\hline Importance $\times$ Severity $\times$ Preference for Integration & & & & & -.30 & .74 & .232 \\
\hline
\end{tabular}

Regression coefficients are from Step 4 of the model, and all variables were centered around their means. $\chi^{2}$ values refer to changes in the -2 Loglikelihood value. Nagelkerke $R^{2}$ values are reported for effect size. ${ }^{\text {a }}$ $p$ values refer to the change between models for the rows labeled Step 1, Step 2, etc 


\section{Anger}

In the first step of the analysis we entered the number of hours participants typically worked. This step was significant, $\chi^{2}=5.43, p=.02, R^{2}=.04$, such that individuals who reported working more hours were less likely to report anger at the interruption. In the second step we entered Importance, Severity, and Preference for Integration. This step added to prediction, $\chi^{2}=53.84$, $p<.001, \Delta \chi^{2}=48.41, p<.001, \Delta R^{2}=.28$. Hypotheses 1,2 , and 4 were supported. The likelihood of reporting anger was positively related to the severity of the interruption, negatively related to the interruptions' importance, and negatively related to one's preference for integrating.

In Step 3 we entered the Importance $\times$ Severity interaction, which was significant, $\chi^{2}=58.92, p<.001, \Delta \chi^{2}=5.08, p=.02, \Delta R^{2}=.03$. As shown in Fig. 1 (graphing values one standard deviation above and below the means of the predictors), Hypothesis 3 was supported. The severity of the interruption was more likely to result in anger when the interruption was unimportant.

To test Hypothesis 5, we added two additional steps to the logistic regression. In Step 4 we added the two-way interactions between Preference for Integration and the features of the interruption. This step did not improve the prediction of anger, $\chi^{2}=59.36, p<.001, \Delta \chi^{2}=.44, p=.80, \Delta R^{2}<.01$. Finally, in Step 5 we added the three-way interaction, which was also not significant, $\chi^{2}=59.663, p<.001, \Delta \chi^{2}=.31, p=.58, \Delta R^{2}<.01$. Thus, Hypothesis 5 was not supported for anger.

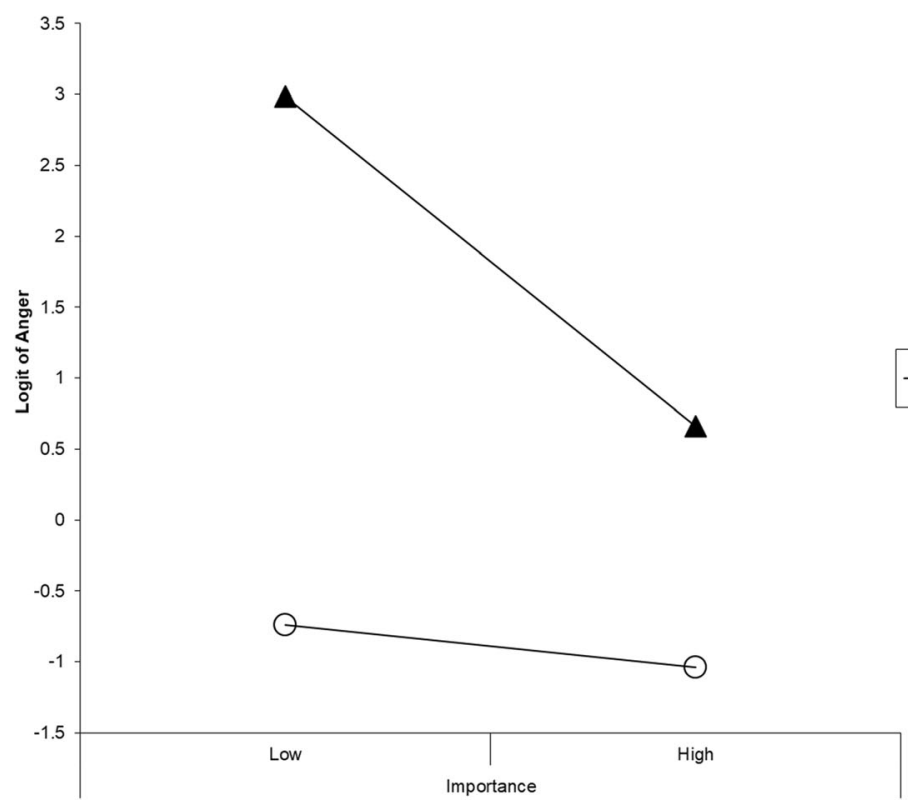

- Severity Low $\rightarrow-$ Severity High

Fig. 1 Anger as a function of interruption importance and severity, at mean levels of hours worked and preference for integration 


\section{Sadness}

In Step 1 the main effects of our predictor variables significantly explained Sadness, $\chi^{2}=40.01, p<.001, R^{2}=.25$. Hypothesis 2 was supported; more severe interruptions were more likely to generate sadness. However, neither Importance nor Preference for Integration were significantly related to Sadness. Therefore, Hypotheses and 1 and 4 were not supported.

In Step 2 the Importance $\times$ Severity interaction approached significance, $\chi^{2}=43.54$, $p<.001, \Delta \chi^{2}=3.52, p=.06, \Delta R^{2}=.02$ (note that in Table 3 this effect appears to be significant due to the inclusion of terms in future steps of the model). Figure 2 shows results similar to that for Anger. Given that this result was not quite significant, it lends partial support for Hypothesis 3.

Similar to the results for Anger, Steps 3 and 4 (adding 2-way interactions and the predicted 3-way interaction, respectively) did not add to the prediction of Sadness; Hypothesis 5 was not supported.

\section{Complaining}

The results for Step $1, \chi^{2}=42.87, p<.001, R^{2}=.26$, showed that Complaining was negatively related to Importance, positively to Severity, and not significantly related to Preference for Integration. Step 2 provided support for Hypothesis $3, \chi^{2}=53.74$, $p<.001, \Delta \chi^{2}=10.86, p<.001, \Delta R^{2}=.06$. Figure 3 shows that the effect of Severity is much stronger for unimportant interruptions.

In Step 3 we entered the two-way interactions between Preference for Integration and the features of the interruption. This step approached significance, $\chi^{2}=59.053, p<.001, \Delta \chi^{2}=$

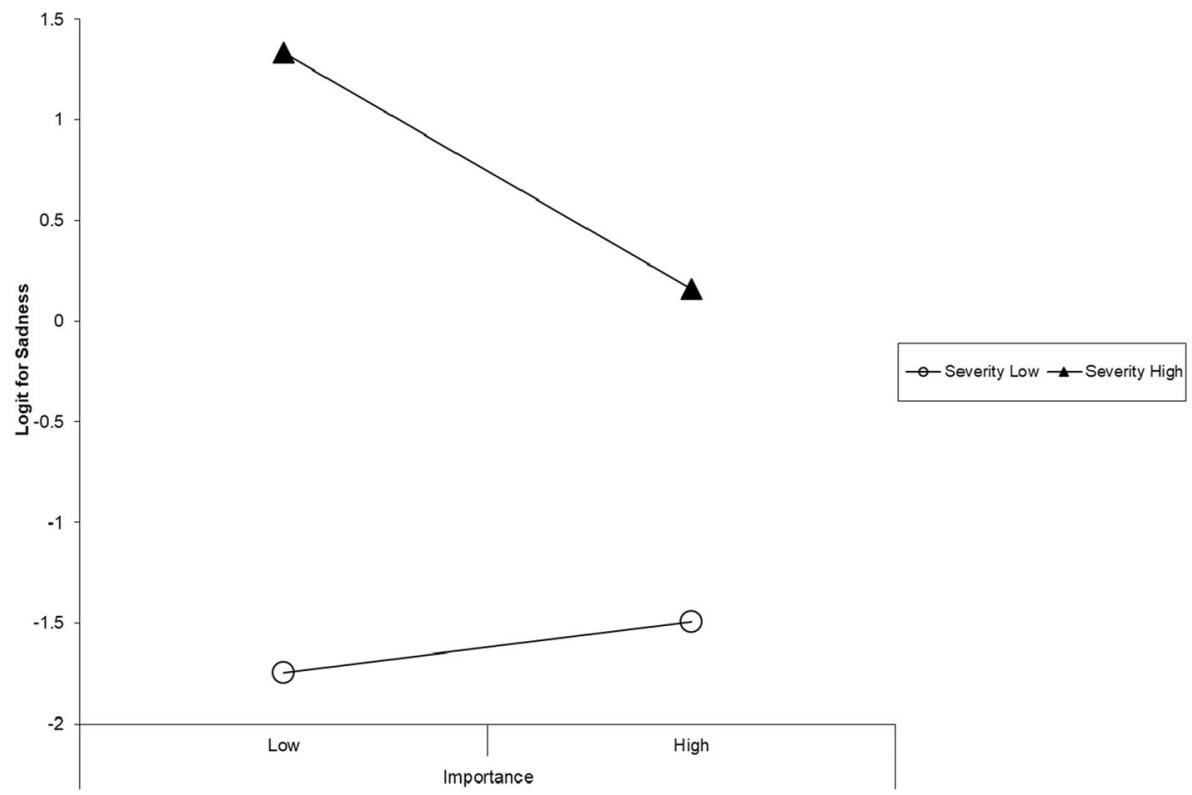

Fig. 2 Sadness as a function of interruption importance and severity, at mean levels of preference for integration 


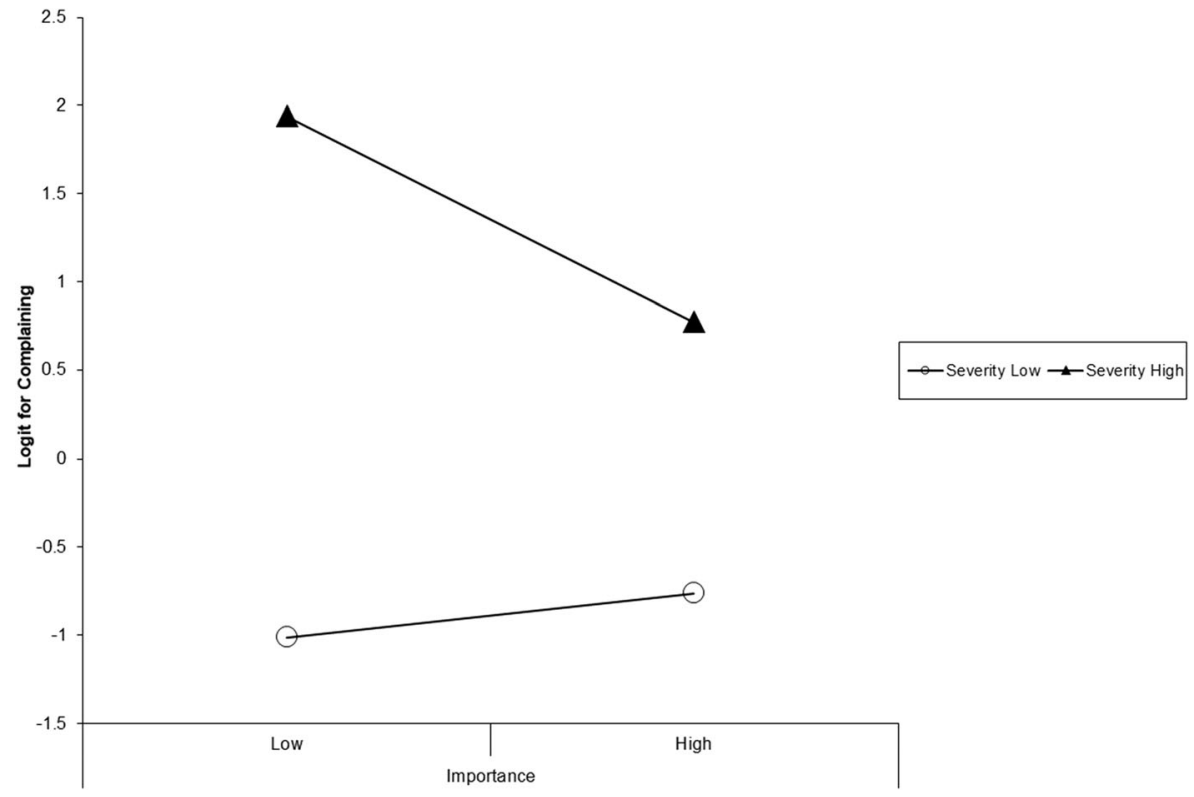

Fig. 3 Complaining as a function of interruption importance and severity, at mean levels of preference for integration

$5.318, p=.07, \Delta R^{2}=.03$. In this step the Severity $\times$ Preference for Integration interaction was significant, $B=.57, p=.03$. As shown in Fig. 4 , a preference for integration was positively related to complaining for severe interruptions, but negatively related for interruptions that

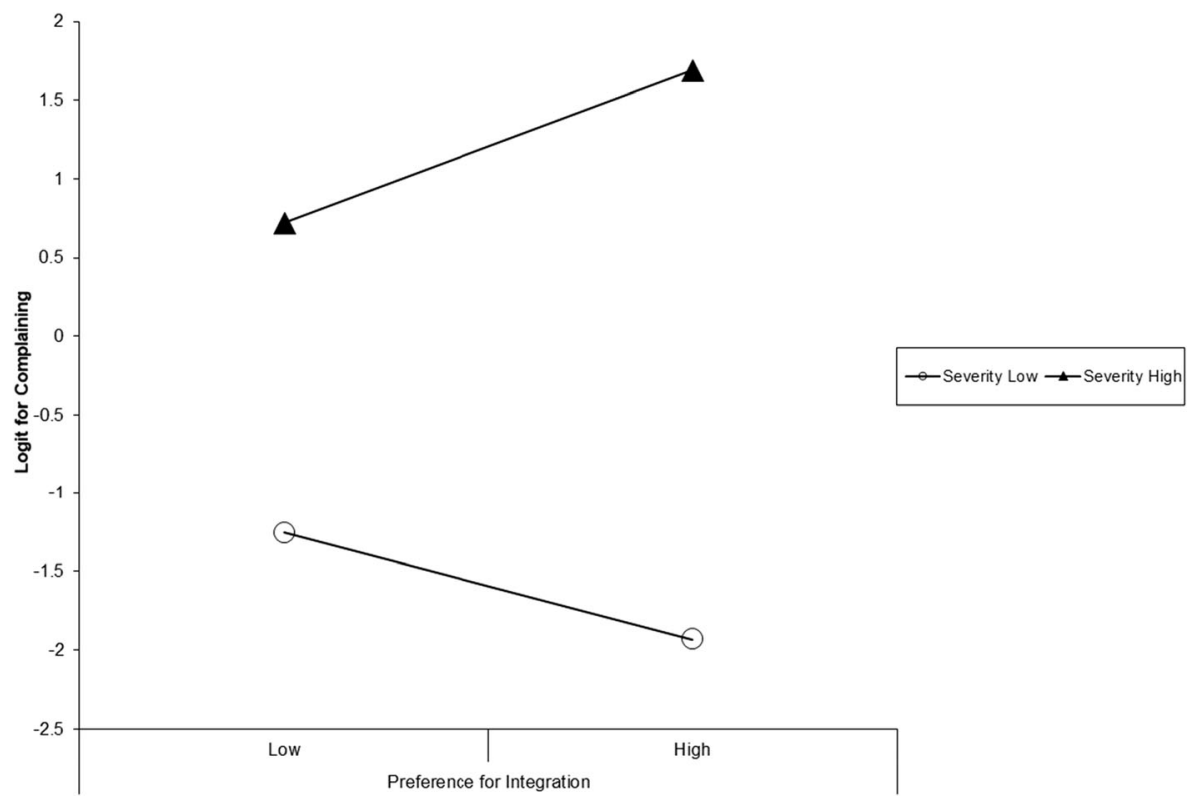

Fig. 4 Complaining as a function of interruption severity and preference for integration, at mean levels of severity 
were not as severe. This interaction was not hypothesized and only approached significance, so it should be interpreted with caution. Finally, Step 4 did not add to the prediction of complaining; Hypothesis 5 was not supported.

\section{Forgiveness}

Step 1 paralleled the results of Complaining, $\chi^{2}=16.81, p<.001, R^{2}=.11$. Hypotheses 1 and 2 (Importance and Severity) were supported, but Hypothesis 4 (Preference for Integration) was not. Similarly, the interaction between Importance and Severity was significant, $\chi^{2}=20.85, p<.001, \Delta \chi^{2}=4.04, p=.04, \Delta R^{2}=.02$. Figure 5 shows that the effect of Severity is more pronounced and the interruption was unimportant, thus supporting Hypothesis 3. However, like the other dependent variables, the three-way interaction for Hypothesis 5 was not supported.

\section{Discussion}

We found mixed results for our hypotheses. The first two hypotheses had consistent support. With one exception (Sadness), the importance of the interruption related to more positive reactions, suggesting that individuals do understand that particular interruptions may be necessary (or, and others may not be). Similarly, across all four dependent variables, interruptions that posed a more substantial disruption to the workday generated more negative reactions.

For three of the reactions these effects were substantial, explaining about a quarter of the variability in reactions (along with boundary management preferences, which did

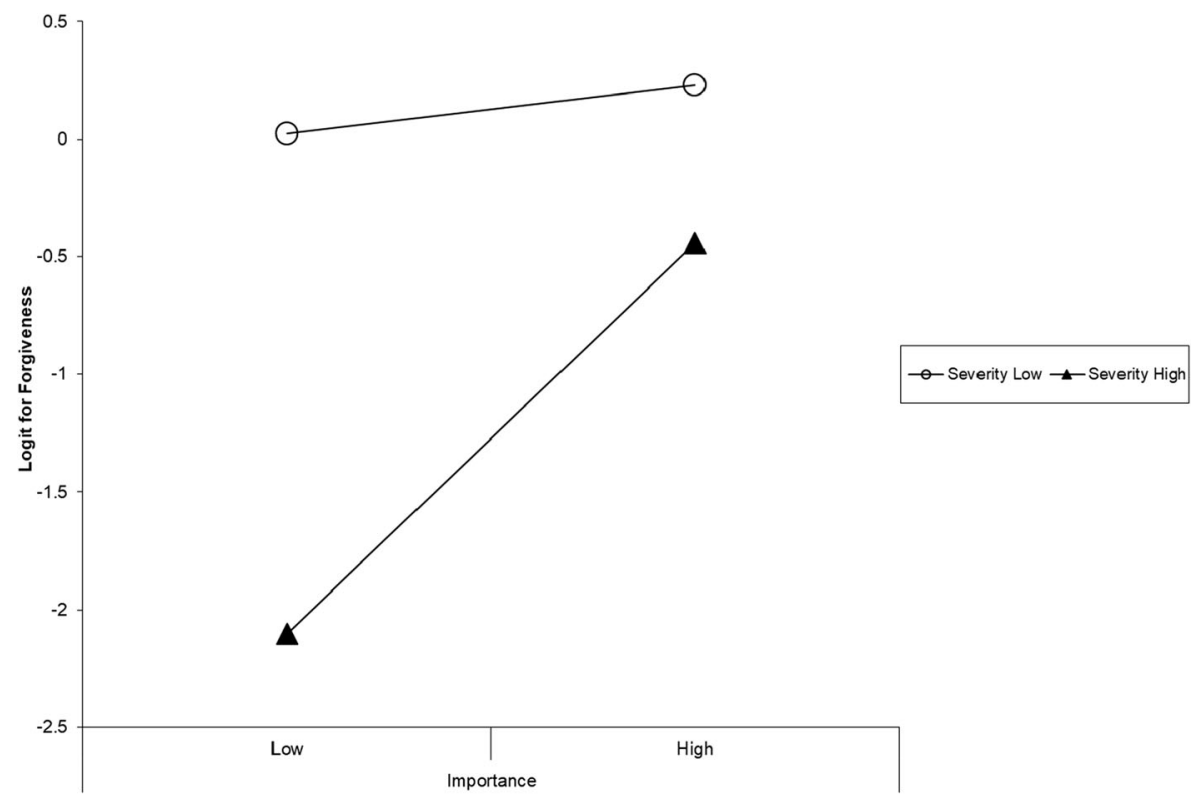

Fig. 5 Forgiveness as a function of interruption importance and severity, at mean levels of preference for integration 
not relate strongly to most reactions). We have thus found strong evidence that the type of interruption matters. An examination of Table 1 (using tests of dependent correlations, Lee \& Preacher, 2013) shows that Severity has stronger direct relationships than Importance for Anger, Sadness, and Complaining. Indeed, Importance did not have significant direct relationships with the dichotomized DV's; its effect only became significant in the logistic regressions after the effects of Severity and Preference for Integration had been controlled for. Given the low correlations among the predictors, we do not believe that multicollinearity can account for Importance's significant effects. Rather, we think it likely that controlling for these other predictors allowed for this effect to emerge. Furthermore, as we explain later, the role of importance is not limited to its direct relationships with the DV's.

The effect size for forgiveness was about half that of the other three reactions. We encourage future research to replicate and explore possible reasons for this relatively weaker effect. For instance, typically close ties with many family members may tend to engender forgiveness regardless of circumstances, leading to restricted range in this variable. The relatively lower reliability of this variable may also contribute to this difference.

We also found consistent support for Hypothesis 3. As shown in the figures, interruptions that were viewed as important ameliorate the effect of severity. The variance explained by this interaction was relatively smaller than the main effects, but they do provide support for the importance of interruption characteristics. Our results show that not all interruptions generate negative reactions. Indeed, interruptions from family that present minor disruptions are not generally seen as aversive. Furthermore, even severe interruptions are less likely to generate negative reactions when there is a good reason for them. Our finding is consistent with arguments made by researchers such as Hunter et al. (2019) that employees focus on satisfying goals in multiple domains, and interruptions at work may be seen as a legitimate way to satisfy those goals.

We found less support for hypotheses related to boundary management preferences (H4 and H5). Individuals who prefer integration did report less anger, but preferences did not relate to the other three outcomes. Furthermore, preferences did not qualify the Severity $\times$ Importance interaction. It is possible that our preferences items, which were related to general tendencies, might not relate to discrete events such as the ones we studied. Instead, such a measure might be better suited for aggregate interruptions and reactions. We encourage future research to measure such preferences and expectations at a daily level.

Although we did not hypothesize it, we found an interaction between preferences and the severity of the interruption for Complaining (see Fig. 4). Individuals who prefer integration seemed to complain less about low-disruption interruptions than those who preferred segmentation. However, they were more likely to complain about severe interruptions. We found this result counterintuitive, but we speculate that individuals who prefer integration may have greater expectations that family members do not take advantage of their openness, or perhaps such individuals experience more frequent interruptions, making a severe interruption particularly frustrating. Given the nature of this finding and the fact that we did not find it consistently across all outcomes, we recommend caution in interpreting this interaction and encourage replication. 
Based on these results, our study makes several important contributions to the literature. First, we add to the small but growing body of research examining discrete instances of boundary violations in the workplace. Specifically, we respond to calls to extend the range of reactions studied (e.g., Hunter et al., 2019; Newton et al., 2020), adding anger, sadness, complaining, and forgiveness to outcomes such as negative affect and perceived goal obstruction. We thus show that boundary violations may generate a range of complex reactions, providing many potential mediating avenues for boundary violations to aggregate into perceptions of WFC. Second, although our results were not as strong, by focusing on preferences for boundary management we contribute to our understanding of the role individual differences play in WFC perceptions. We address a third gap in the literature (e.g., Pachler et al., 2018; Wilkes et al., 2018) by showing that not all interruptions are the same - characteristics of boundary violations affect reactions and interact with each other to exacerbate or ameliorate such reactions. In addition to building on other typologies of interruptions (e.g., Jett \& George, 2003), these results also illustrate applications of other theories. For instance, severity's effect is consistent with theories such as Conservation of Resources Theory (Hobfoll, 1989). As time is a limited resource, interruptions that deplete one's temporal resources are likely to generate negative reactions. Furthermore, the effect of importance supports theories such of multiple goal self-regulation (Sun \& Frese, 2013), showing how individuals balance satisfying family and work goals.

\section{Future Research Directions and Limitations}

As the research on episodic boundary interruptions is novel, directions for future research abound and we discuss only a few here. First, we measured only a small subset of possible outcomes. In addition to anger, sadness, complaints, and forgiveness, future research should add workplace variables such as perceived changes in workload (e.g., rearranging work as a result of the interruption) or subsequent job performance. Exploring mediators and potential moderators of these relationships would also be fruitful. For instance, Newton et al.'s (2020) concept of attention residue suggests that rumination about the interruption might affect subsequent task performance, indicating the need to measure rumination and resource depletion. It would be interesting to see if facets of the interaction (such as whether the reason for the interruption was resolved) altered the effects of the interruption on rumination. Next, although we did not find support for boundary management preferences moderating the effects of the interruption's features, it is possible that other similar constructs (e.g., polychronicity, Pachler et al., 2018) may moderate such relationships.

Additionally, we realize that episodic boundary management research will ultimately need to connect with the more common approach (i.e., that relates typical boundary management to broader outcomes such as general WFC perceptions). Future research needs to explore the mechanisms through which individual episodes of interruptions manifest in outcomes such as overall job or relationship satisfaction. For instance, is a single violation of expectations sufficient to affect more holistic outcomes among those who prefer segmentation, or must a particular threshold be reached?

Next, we recommend expanding beyond the workplace. In addition to workplace outcomes, we expect that the effects of interruptions extend to the family domain, so we recommend extending our research to employee and family members' interactions after 
work (e.g., marital satisfaction; quality of relationships with family). Next, by focusing on interruptions from family into work we neglected potentially parallel effects of work interruptions into family or personal time. Similarly, by specifically asking for instances of interruptions, we limited our study of boundary violations in a single direction, as only those who prefer segmentation could perceive a boundary violation. It may be interesting to examine the effects of an absence of interruptions among individuals who desire more frequent contact with family during work.

Our study focused on the negative effects of interruptions (e.g., anger, sadness, complaining and forgiveness that arose from an initially negative reaction). However, interruptions can also be positive. Hunter et al. (2019) discuss how interruptions into one domain may allow for the satisfaction of goals in the other domain. Jett and George (2003) similarly discuss how interruptions may allow for coworker needs to be met, strengthening the organization, and this argument can easily extend to family outcomes. Such interruptions, for instance, could be construed as restorative and enjoyable breaks. We encourage research that explores positive reactions to interruptions and the characteristics that may lead to such positive outcomes.

One of the study's salient limitations is the cross sectional and retrospective nature of the design. As we measured all variables at the same time, and as all were based on recall of a past event, it is difficult to establish causality. For instance, individuals who were angry at the time of the interruption may have consequently judged the severity of the incident more negatively. Alternatively, individuals' negative affectivity may have led to both anger and more negative judgments. Variables such as the degree of affection for the family member may simultaneously influence perceived judgments of the interruption or even boundary management preferences. Given the nature of the variables under study it may be difficult to employ an experimental design, but future research may consider techniques such as experience sampling.

One limitation of our study is that we created several of the measures that we used. Although we employed some content validity and convergent validity strategies to improve the measures and assure their quality, our results still may not generalize to other measures. In particular, our measure of forgiveness had low reliability and effect sizes lower than those for the other dependent variables, suggesting the need for an improved measure. We recommend that future research replicate our results with other measures.

The source of the data (Mechanical Turk) may be another limitation of the study. As reviewed by Walter et al. (2019), questions have been raised regarding participant honesty, representativeness, and other factors. However, there is also evidence supporting results drawn from online panel studies. For instance, Walter et al.'s meta-analysis showed minimal evidence or differences in reliabilities or effect sizes between online panel and traditional samples. Nevertheless, our results should be replicated in different samples.

A related concern is the number of individuals we screened out prior to our analyses. One reason that we did so is the concern that automated computer programs were masquerading as participants around the time of our first sample (e.g., Dreyfuss, 2018; Moss \& Litman, n.d.a). Subsequent research suggested that the decrease in data quality around that time may be attributable to server farms that allowed individuals outside the United States to participate in studies that were supposed to be limited to US participants (Moss \& Litman, n.d.b). 
Regardless of the source, we observed that many individuals were not paying sufficient attention. For instance, while many participants correctly responded when asked to provide a qualitative description of a specific incident of interruption, some talked about interruptions in general, whereas others responded with nonsensical answers such as "Talk a little bit about what your current role is, the scope of it, and perhaps a big recent accomplishment." If we were to have included these individuals the quality of our results would have been questionable. As support for our practice, we draw on researchers such as Thomas and Clifford (2017). Their review of the use of screener questions showed that failure rates can be as high as 52\%; our study (46\%) is not out of the ordinary. Furthermore, they found that screening out low-quality respondents can improve the power of the study, with limited effects on the external validity of the results.

\section{Practical Implications}

The results of our study suggest multiple ways to ameliorate the effects of specific interruptions and improve workplace functioning. First, we echo Pachler et al.'s (2018) recommendation to educate employees and managers about the effects of interruptions - in the present case, effects on anger, sadness, complaining, and forgiveness. The particularly unique effect of unimportant interruptions that generate substantial disruptions should be highlighted.

Relatedly, employees could be trained to compensate for the effects of such interruptions. For instance, assuming that one reason for negative reactions to severe interruptions is the likelihood that it is affecting performance, employees could be taught to implement strategies that may help them resume their task (e.g., making a quick note about where they were in the task before addressing the interruption; generating implementation intentions or jotting down a reminder to complete the task later). Additionally, training could include strategies to deal with the emotional reactions generated by the interruption (e.g., taking a moment to calm down; actively forgiving the person who interrupted; choosing not to engage in work tasks on which one's negative affect would have an effect).

Finally, employees and managers should work to find ways to eliminate severe and unimportant interruptions. Policies (personal or organizational) that prohibit all interruptions may work, although such policies may be perceived as unfair and may block necessary communications. Instead, employees could explicitly converse with their family members (cf. Kreiner et al., 2009) about their expectations, relying on these "border keepers" (Clark, 2000) to help protect the boundaries between home and work lives. Furthermore, in some workplaces it may be possible to structure one's day to permit or encourage family communications at certain times (e.g., Hunter et al., 2019; Pachler et al., 2018). Employees may be able to structure their days so that during these times they are not working on complex tasks to which interruptions would be particularly disruptive.

Funding Not applicable.

Data Availability Upon request. 
Code Availability Upon request.

\section{Declarations}

Ethics Approval Obtained from the University's IRB.

Consent for Publication All listed authors consent to publication.

Consent to Participate Given.

Conflict of Interest On behalf of all authors, the corresponding author states that there is no conflict of interest.

\section{References}

Allen, T. D., French, K. A., Dumani, S., \& Shockley, K. M. (2020). A cross-national meta-analytic examination of predictors and outcomes associated with work-family conflict. Journal of Applied Psychology, 105, 539-576. https://doi.org/10.1037/ap10000442.

Ashforth, B. E., Kreiner, G. E., \& Fugate, M. (2000). All in a day's work: Boundaries and mirco role transitions. Academy of Management Review, 25, 472-491.

Baethge, A., \& Rigotti, T. (2013). Interruptions to workflow: Their relationships with irritation and satisfaction with performance, and the mediating roles have time pressure and mental demands. Work \& Stress: An International Journal of Work, Health and Organisations, 27, 43-63.

Beal, D. J., Weiss, H. M., Barros, E., \& MacDermid, S. M. (2005). An episodic process model of affective influences on performance. Journal of Applied Psychology, 90, 1054-1068.

Carver, C. S., Scheier, M. F., \& Weintraub, J. K. (1989). Assessing coping strategies: A theoretically based approach. Journal of Personality and Social Psychology, 56, 267-283.

Clark, S. C. (2000). Work/family border theory: A new theory of work/family balance. Human Relations, 53, 747-770.

Dreyfuss, E. (2018). A bot panic hits Amazon's Mechanical Turk. Wired. https://www.wired.com/story/ amazon-mechanical-turk-bot-panic/. Accessed 22 Feb 2021.

Frone, M. R., Russell, M., \& Cooper, M. L. (1992). Antecedents and outcomes of work-family conflict: Testing a model of the work-family interface. Journal of Applied Psychology, 77, 65-78.

Greenhaus, J. H., \& Beutell, N. J. (1985). Sources of conflict between work and family roles. Academy of Management Review, 10, 76-88.

Hobfoll, S. E. (1989). Conservation of resources: A new attempt at conceptualizing stress. American Psychologist, 44, 513-524.

Hunter, E. M., Clark, M. A., \& Carlson, D. S. (2019). Violating work-family boundaries: Reactions to interruptions at work and home. Journal of Management, 45, 1284-1308.

Jett, Q. R., \& George, J. M. (2003). Work interrupted: A closer look at the role of interruptions in organizational life. Academy of Management Review, 28(3), 494-507.

Kossek, E., \& Lautsch, B. (2012). Work-family boundary management styles in organizations: A cross-level model. Organizational Psychology Review, 2, 152-171. https://doi.org/10.1177/204138661143626.

Kossek, E. E., Ruderman, M. N., Braddy, P. W., \& Hannum, K. M. (2012). Work- Nonwork boundary management profiles: A person-centered approach. Journal of Vocational Behavior, 81, 112-128.

Kreiner, G. E., Hollensbe, E. C., \& Sheep, M. L. (2009). Balancing borders and bridges: Negotiating the workhome interface via boundary work tactics. Academy of Management Journal, 52, 704-730.

Lazarus, R. S. (1966). Psychological stress and the coping process. New York: McGraw-Hill.

Lee, B. C., \& Duffy, V. G. (2015). The effects of task interruption on human performance; a study of the systematic classification of human behavior and interruption frequency. Human Factors and Ergonomics in Manufacturing \& Service Industries, 25(2), 137-152. https://doi.org/10.1002/hfm.20603.

Lee, I, A., \& Preacher, K, J. (2013). Calculation of the test of the difference between two dependent correlations with one variable in common [Computer software]. Available from http://quantpsy.org. Accessed 22 Feb 2021. 
Madsen, S. R., Gygi, J., Hammond, S. C., \& Plowman, S. F. (2009). Forgiveness as a workplace intervention: The literature and a proposed framework. Journal of Behavioral and Applied Management, 10, 246-262.

Michel, J. S., Mitchelson, J. K., Kotrba, L. M., LeBreton, J. M., \& Baltes, B. B. (2009). A comparative test of work-family conflict models and critical examination of work-family linkages. Journal of Vocational Behavior, 74, 199-218.

Moss, A., \& Litman, L. (n.d.a). Concerns about bots on mechanical Turk: Problems and solutions. CloudResearch. https://www.cloudresearch.com/resources/blog/concerns-about-bots-on-mechanicalturk-problems-and-solutions/. Accessed 22 Feb 2021.

Moss, A., \& Litman, L. (n.d.b). After the bot scare: Understanding what's been happening with data collection on MTurk and how to stop it. CloudResearch. https:/www.cloudresearch.com/resources/blog/concernsabout-bots-on-mechanical-turk-problems-and-solutions/. Accessed 22 Feb 2021.

Newton, D. W., Le Pine, J. A., Kim, J. K., Wellman, N., \& Bush, J. T. (2020). Taking engagement to task: The nature and functioning of task engagement across transitions. Journal of Applied Psychology, 105, 118. https://doi.org/10.1037/ap10000428.

Nippert-Eng, C. E. (1996). Home and work. Chicago, IL: The University of Chicago Press.

Nohe, C., Meier, L. L., Sonntag, K., \& Michel, A. (2015). The chicken or the egg? A meta-analysis of panel studies of the relationship between work-family conflict and strain. Journal of Applied Psychology, 100, $522-536$.

Pachler, D., Kuonath, A., Specht, J., Kennecke, S., Agthe, M., \& Frey, D. (2018). Workflow interruptions and employee work outcomes: The moderating role of polychronicity. Journal of Occupational Health Psychology, 3, 417-427.

Riek, B. M., \& Mania, E. W. (2012). The antecedents and consequences of interpersonal forgiveness: A metaanalytic review. Personal Relationships, 19, 304-325.

Schmidt, A. M., Dolis, C. M., \& Tolli, A. P. (2009). A matter of time: Individual differences, contextual dynamics, and goal progress effects on multiple-goal self-regulation. Journal of Applied Psychology, 94, $692-709$.

Smit, B. W., Maloney, P. W., Maertz Jr., C. P., \& Montag-Smit, T. (2016). Out of sight, out of mind? How and when cognitive role transition episodes influence employee performance. Human Relations, 69(11), 2141-2168. https://doi.org/10.1177/0018726716636204.

Sun, S. H., \& Frese, M. (2013). Multiple goal pursuit. In E. A. Locke \& G. P. Latham (Eds.), New developments in goal setting and task performance (pp. 177-194). New York: Routledge.

Thomas, K. A., \& Clifford, S. (2017). Validity and mechanical Turk: An assessment of exclusion methods and interactive experiments. Computers in Human Behavior, 77, 184-197. https://doi.org/10.1016/j.chb.2017. 08.038 .

Viswesvaran, C., Sanchez, J. I., \& Fisher, J. (1999). The role of social support in the process of work stress: A meta-analysis. Journal of Vocational Behavior, 54, 314-334.

Walter, W. L., Seibert, S. E., Goering, D., \& O’Boyle Jr., E. H. (2019). A tale of two sample sources: Do results from online panel data and conventional data converge? Journal of Business and Psychology, 34, 425-452. https://doi.org/10.1007/s10869-018-9552-y.

Watson, D., \& Clark, L, A. (1994). The PANAS-X: Manual for the Positive and Negative Affect scale Expanded Form. Downloaded from https://www2.psychology.uiowa.edu/faculty/clark/panas-X.pdf. Accessed 22 Feb 2021.

Wilkes, S. M., Barber, L. K., \& Rogers, A. P. (2018). Development and validation of the workplace interruptions measure. Stress and Health, 34, 102-114.

Publisher's Note Springer Nature remains neutral with regard to jurisdictional claims in published maps and institutional affiliations. 